\title{
SHAPE PRESERVING NOISE ATTENUATION MODEL FOR 3-D-MODELING OF HERITAGE SITES BY PORTABLE LASER SCANS
}

\author{
Tian Zhang, Joelle Abu-Hani, Sagi Filin \\ Mapping and Geo-Information Engineering, Technion - Israel Institute of Technology, Haifa, Israel \\ (tianz, abu.h, filin)@ technion.ac.il
}

\section{Commission II}

KEY WORDS: Point clouds, Noise attenuation, 3-D modeling, Laser scanning, Portable laser scanners, Cultural Heritage

\begin{abstract}
:
The development of efficient strategies to document cultural heritage sites is an active research field. One promising avenue to address that need may be found in the use of portable laser scanners. Such scans provide a mapping-grade level of accuracy, yet their level of characterization is limited by the low resolution of the generated point cloud and by the relatively noisy measurements. In this paper we study methods to attenuate the noisy responses as a means to improve the data quality and highlight the underlying structure. Unlike the prevailing plane-fitting-based filtering approaches that tend to blur salient features, we consider the use of local structure properties in our denoising strategy. We use the normal-based bilateral filtering of point clouds as a platform, yet introduce new normal preservation concepts whose incorporation significantly improves the overall denoising process performance. Results demonstrate how our proposed solution outperforms the standard plane-filtering and the naive bilateral approach. The attenuation we achieve yields a more visually pleasing entity description as well as simplified processing of subsequent procedures, including feature extraction and semantic segmentation.
\end{abstract}

\section{INTRODUCTION}

Three-dimensional documentation of cultural heritage $(\mathrm{CH})$ sites has been receiving increased attention in recent years. This attention stems from the need for detailed documentation of sites in light of damages and deterioration caused by the passage of time, the need for preservation, and the desire for increased accessibility. Thanks to the plethora of data acquisition technologies, detailed and accurate 3 -D point cloud information can be gathered on a large scale, facilitating a diverse set of applications, including historical site interpretation, intervention planning, monitoring, augmented and virtual reality, and generation of heritage building information models (HBIM), as only a few examples (López et al., 2017; Murtiyoso and Grussenmeyer, 2019; Capone and Lanzara, 2019).

The quality of the acquired 3-D point clouds differs according to the scanning platform. For example, terrestrial laser scanners (TLS) are marked by the high level of accuracy and detail they provide. However, their utilization at large-scale sites can be time-consuming and cumbersome, thereby limiting their productivity (Russhakim et al., 2019). In contrast, the utilization of portable laser scanners (PLS), mostly handheld, is characterized by their lower resolution and noisy responses. They facilitate, though, an efficient survey of large-scale sites, making their application attractive (e.g., Russhakim et al., 2019; Patrucco et al., 2019; Bronzino et al., 2019). In that respect, Russhakim et al. (2019) reported that three persons and $60 \mathrm{~min}$. were needed to document a site when using TLS, yet only a single person and $30 \mathrm{~min}$. were required for the same task when using PLS. Patrucco et al. (2019) referred to the ability to achieve a complete site survey using PLS, enabling to cover regions invisible to TLS due to occlusions or lack of accessibility; while Bronzino et al. (2019) alluded to the ability to recognize architectural details of medium-small dimensions, valuable for the

\footnotetext{
* Corresponding author
}

construction of detailed building information models for restoration, management, and maintenance purposes.

The high level of noise, characteristic of PLS, necessitates further processing to facilitate applications such as semantic interpretation, rendering, and architectural modeling (Xie and Wang, 2017; Cui et al., 2019; Wang et al., 2020). Cui et al. (2019) marked the large footprints of portable devices, the registration uncertainty due to the motion of the operators, and the nature of the scanned entities as sources responsible for this high level of noise. Without proper treatment, the authors noted that the use of PLS data may lead to algorithmic failure and poor results in the estimation of geometric primitives. This in turn would affect the semantic interpretation of scenes and 3-D modeling. For instance, the extraction of geometric primitives, vital for segmentation algorithms or surface fitting, suffer from noise effect on the features (Xia et al., 2020; Wang et al., 2020). To demonstrate that, the authors described how line-based 3-D reconstruction approaches suffer from an excessive amount of false details due to noisy responses, thereby affecting the quality of built models (Cui et al., 2019).

To improve the representation and to facilitate additional processing, it is customary to attenuate the noise effect within the point cloud data. Under the assumption of dense structured surfaces, Orts-Escolano et al. (2013) proposed a voxel-based filtering approach. First the data were partitioned by a set of userdefined cells, then ones in which the number of points was below a predefined threshold were filtered. Methods of that kind favor densely sampled regions, hardly retain sparsely sampled entities and lead to loss of information. More recently, planefitting-based approaches have been gaining increased attention. Nurunnabi et al. (2015) proposed a random sample consensus (RanSaC)-based method which estimates the best-fit plane and rejects outliers by minimizing the sum of projected distances. Ning et al. (2018) used a point deviation from a local fitted 
plane as a noise reduction criterion. Such approaches are now widely used, as in the standard procedure in the CloudCompare (v.2.11, 2021). They require an empirical inlier and outlier threshold definition, and more importantly, surface forms that abide by the local planarity assumption. Instead of performing noise removal, Digne and De Franchis (2017) described a bilateral filter approach applied directly on point clouds whose aim was to redistribute points through a weighted averaging while considering proximity and shifting points along the normal direction. Alternative forms have been using the graph Laplacian-based framework for denoising, aimed at promoting the smoothness via minimizations the sum of total variations of features (Zeng et al., 2019; Dinesh et al., 2020). Nonetheless, such approaches tend to degrade sharp transitions and produce artifacts at unevenly sampled regions. To address that, Liu et al. (2020) proposed improved normal-based weighted averaging by using refined orientations, where sharp transitions are firstly identified from low noisy responses then their normals are consolidated by majority voting. Their approach has contributed to a point-wise smooth outcome but did not necessarily conform to global trends, sometimes requiring identification of salient points from the noisy response.

While existing research into PLS-based $\mathrm{CH}$ documentation has focused mainly on quantifying the data quality, little has been devoted to manners by which the point cloud data of the underlying structures can be enhanced. To address the non-uniform density and high noise level of data of that kind, we present in this paper a shape-preserving denoising model that improves the representation of architectural structures in heritage sites. We propose a normal-based strategy as a means to follow the locally structured characteristics of architectural elements. As a framework we use the normal-based bilateral filtering, where denoising is approached according to the local point orientation. This choice is motivated by its local application and computational efficiency. We show, however, that by using a refined definition of the surface normals, the shape preservation problem is better addressed. Results demonstrate how our solution outperforms the standard plane-filtering methods as well as the naive bilateral approach. We show that in contrast to such approaches that obscure salient features, vital to the presentation of the site, ours preserves them yet attenuates the noise effect. Improvement of the point cloud data quality paves the way to a more visually pleasing site presentation as well as to simplified subsequent processing.

\section{METHODOLOGY}

Standard noise attenuation approaches are plane-fitting based. They consider the distance of all points in a given neighborhood to the underlying surface. To do so, they locally fit a plane around each point $\mathbf{x}_{i}$, then remove a neighbor point, $\mathbf{x}_{j} \in N\left(\mathbf{x}_{i}\right)$, if its projected distance, $d$, along surface normal $\mathbf{n}_{i}$ is larger than the average distance $\bar{d}$ plus the standard deviation $\sigma_{p}$ (e.g., Ning et al., 2018).

$$
d=\left\langle\mathbf{n}_{i}, \mathbf{x}_{j}-\mathbf{x}_{i}\right\rangle
$$

While successful when the underlying surface is (locally) planar and is also distinguishable, the unimodality of the surface form is an underlying assumption, and the quality and correctness of the normal estimation is pivotal. Also, as they are based on the minimization of squared distances and require data of low noise level, they act as cleaning solution (Sanchez et al., 2020) limiting their application to portable laser scans.
To overcome the limitations associated with such denoising forms, we propose a local surface attentive solution. Realizing that surface normals capture the local entity's form, they should play a pivotal role in denoising the cloud. Therefore, we focus first on their computation and propose a formulation that accommodates the surface variations. As our starting point, we use the iterative reweighted minimization least-squares (IRLS) method. We compute the eigenvector associated with the smallest eigenvalue of a weighted tensor form. For the set of normalized weights, $\left\{w_{j}\right\}_{j=0, \cdots, n}$, associated with the neighbors of a point $\mathbf{x}_{i}$, the weighted tensor is defined by Eq. (2):

$$
\mathbf{T}=\frac{1}{n} \sum_{j}^{n} w_{j}\left(\mathbf{x}_{j}-\overline{\mathbf{x}}\right) \otimes\left(\mathbf{x}_{j}-\overline{\mathbf{x}}\right)
$$

where $\otimes$ is the outer product, $\mathbf{x}_{j}$ is 3 -D neighboring point, $j=1, \cdots, n$, and $\overline{\mathbf{x}}$ is the centroid of the set of points (the mean of neighbor points). Defining $\delta_{j, i}=\mathbf{x}_{j}-\mathbf{x}_{i}$, and $r_{j}=\left\langle\mathbf{v}_{3}, \delta_{j, i}\right\rangle$ the projected distances of $\delta_{j, i}$ on $\mathbf{v}_{3}$, the eigenvector associated with the smallest eigenvalue, we compute (Sanchez et al., 2020):

$$
W_{j}=\left(\frac{\eta}{\eta+r_{j}^{2}}\right)^{2}
$$

and $w_{j}$ :

$$
w_{j}=\frac{W_{j}}{\sum_{k=1}^{n} W_{k}}
$$

where $\eta$ is firstly initialized as the maximum of squared projection distances and decreases at each iteration to favor smaller inlier residuals. Unlike the plane-fitting-based filtering model, this process is iterative, where we constantly identify outliers and eliminate their effect by adjusting their weights. For speedup, the principal components of $\mathbf{T}$, given by the eigenvalues and eigenvectors, $\lambda_{j}$ and $\mathbf{v}_{j}$, such that:

$$
\mathbf{T}_{\mathbf{j}}=\lambda_{j} \mathbf{v}_{\mathbf{j}}, \quad j \in\{1,2,3\}
$$

are computed in a closed-form rather than applying a general decomposition of the roots. Defining the characteristic polynomial $a \lambda^{3}+b \lambda^{2}+c \lambda+d=0$, the eigenvalues are computed as follows (Kopp, 2008):

$$
\begin{aligned}
& \lambda_{1}=\mu+2 \nu \cdot \cos \theta \\
& \lambda_{2}=\mu+2 \nu \cdot \cos \theta+120^{\circ} \\
& \lambda_{3}=3 \mu-\lambda_{1}-\lambda_{2}
\end{aligned}
$$

where:

$$
\begin{aligned}
\mu & =\frac{\operatorname{tr}(\mathbf{T})}{3} \\
\nu & =\sqrt{\frac{\operatorname{tr}\left((\mu \cdot \mathbf{I}-\mathbf{T})^{2}\right)}{6}} \\
\theta & =\frac{1}{3} \cos ^{-1}\left(\frac{1}{2} \operatorname{det}(\mathbf{T}-\mu \mathbf{I})\right)
\end{aligned}
$$

and $\mathbf{I}$ is a $3 \times 3$ identity matrix. The relevant eigenvector $\mathbf{v}_{3}$ is the surface normal, and is computed by the cross product of any two rows of $\mathbf{T}-\lambda_{3} \mathbf{I}$.

\subsection{Normal field refinement}

By using local point neighborhoods, certain points of other surfaces near sharp edges or corners are inevitable to be introduced 


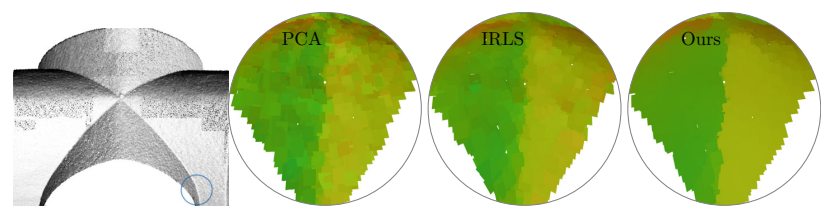

Figure 1. Comparison of normal estimation along a groin vault arris using PCA and IRLS and our method. Points are colored by their normal orientations.

as neighbors for a studied point. Having refined their original noisy form, we turn to improve their estimation by introducing an anisotropic neighborhood, as in the framework of bilateral normal smoothing (Huang et al., 2013). Instead of searching for salient points from highly noisy responses and performing a majority voting (Liu et al., 2020), we consider an implicit manner to consolidate normals at sharp transitions without identifying them. For a point $\mathbf{x}_{i}$, our aim is to minimize the difference between its assigned normal $\mathbf{n}_{\mathbf{i}}$ and other normals $\mathbf{n}_{\mathbf{j}}$ in its neighborhood $\mathbf{x}_{j} \in N\left(\mathbf{x}_{i}\right)$. This can be cast as an optimization problem of the form:

$$
\left.\underset{\mathbf{n}_{\mathbf{i}}}{\operatorname{argmin}}\left\|\mathbf{n}_{\mathbf{i}}-\mathbf{n}_{\mathbf{j}}\right\|^{2} w_{d}\left(\left\|\delta_{j, i}\right\|\right)\right) \phi\left(\mathbf{n}_{\mathbf{i}}, \mathbf{n}_{\mathbf{j}}\right)
$$

where the normal weight function is defined as:

$$
\phi\left(\mathbf{n}_{\mathbf{i}}, \mathbf{n}_{\mathbf{j}}\right)=\exp \left(-\left(\frac{1-\mathbf{n}_{\mathbf{i}}^{\mathbf{T}} \mathbf{n}_{\mathbf{j}}}{1-\cos \left(\sigma_{n}\right)}\right)^{2}\right)
$$

and where the angular parameter, $\sigma_{n}$, scales the similarity of the neighboring normals. This local optimization can be solved by iteratively updating $\mathbf{n}_{\mathbf{i}}$ as follows:

$$
\mathbf{n}_{\mathbf{i}} \leftarrow \frac{\left.\sum_{\mathbf{x}_{j} \in N\left(\mathbf{x}_{i}\right)} w_{d}\left(\left\|\delta_{j, i}\right\|\right)\right) \phi\left(\mathbf{n}_{\mathbf{i}}, \mathbf{n}_{\mathbf{j}}\right) \mathbf{n}_{\mathbf{j}}}{\left.\sum_{\mathbf{x}_{j} \in N\left(\mathbf{x}_{i}\right)} w_{d}\left(\| \delta_{j, i}||\right)\right) \phi\left(\mathbf{n}_{\mathbf{i}}, \mathbf{n}_{\mathbf{j}}\right)}
$$

where $w_{d}\left(\left\|\delta_{j, i}\right\|\right)=\exp \left(-\left\|\delta_{j, i}\right\|^{2} / \sigma_{r}^{2}\right)$ is a decreasing function, and $\sigma_{r}$ is the noise bandwidth. Eq. (10) allows to distinguish normals across discontinuities, and classify their directions into disjoint clusters near each sharp edge due to the high variance values of the PCA normals in that vicinity. Therefore, normal ambiguity at sharp edges is resolved to better represent the underlying geometry as shown in Fig. (1).

\subsection{Denoising}

With the improved quality of the surface normals and their adherence to the underlying surface shape, it is possible to improve the local plane-based denoising by also considering the surface normals as an attribute. Our proposed method follows the idea of preserving normals proposed by bilateral filtering for point clouds (Digne and De Franchis, 2017) were a point $\mathbf{x}_{i}$ is rearranged given its normal $\mathbf{n}_{i}$ to:

$$
\mathbf{x}_{i}+\mathbf{d} \mathbf{x}_{i} \cdot \mathbf{n}_{\mathbf{i}}
$$

The motion per point, $\mathbf{d} \mathbf{x}_{i}$, is a weighted averaging form as shown in Fig. (2) considering Euclidean proximity and normal similarity,

$$
\mathbf{d x}_{i}=\frac{\sum_{\mathbf{x}_{j} \in N\left(\mathbf{x}_{i}\right)} w_{d}\left(|| \delta_{j, i}||\right) w_{n}\left(\left|\left\langle\mathbf{n}_{i}, \delta_{j, i}\right\rangle\right|\right)\left\langle\mathbf{n}_{i}, \delta_{j, i}\right\rangle}{\sum_{\mathbf{x}_{j} \in N\left(\mathbf{x}_{i}\right)} w_{d}\left(|| \delta_{j, i}||\right) w_{n}\left(\left|\left\langle\mathbf{n}_{i}, \delta_{j, i}\right\rangle\right|\right)}
$$

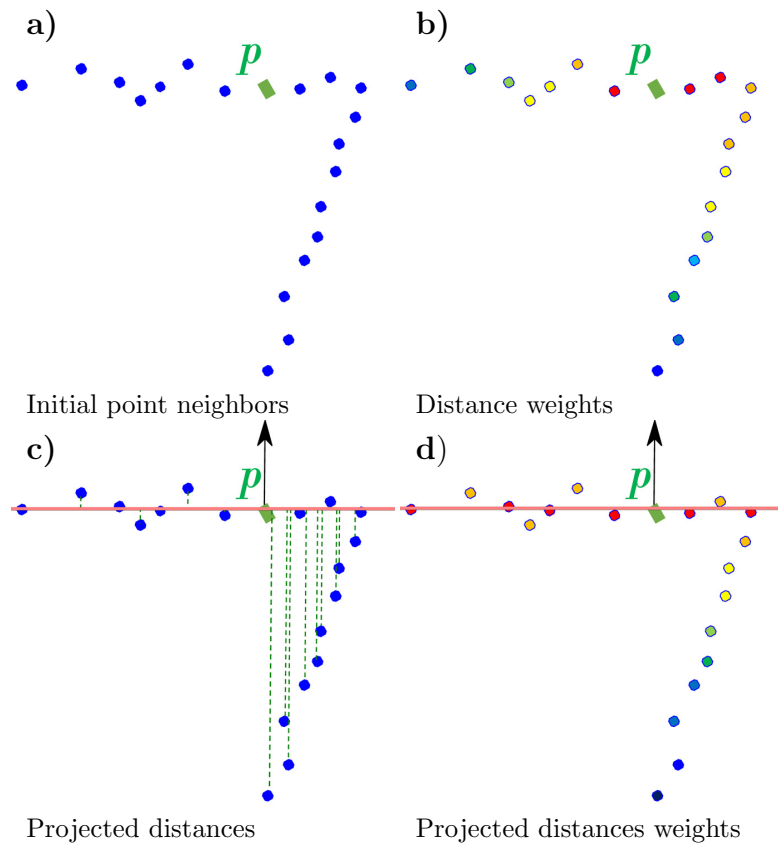

Figure 2. Weighted forms of filtering in $\mathrm{b}, \mathrm{d}$ ) blue $=$ small weights, red $=$ large weights.

where $N\left(\mathbf{x}_{i}\right)$ represents the neighborhood of $\mathbf{x}_{i}$, and $w_{n}\left(\left|\left\langle\mathbf{n}_{i}, \delta_{j, i}\right\rangle\right|\right)=\exp \left(-\left|\left\langle\mathbf{n}_{i}, \delta_{j, i}\right\rangle\right|^{2} / \sigma_{r_{n}}^{2}\right)$ is a decreasing function similar to $w_{d}$, and $\sigma_{r_{n}}$ is the the projected distance bandwidth. These weighted forms favor close coplanar neighboring points while scaling down the effect of distant points and ones of other surfaces.

\section{ANALYSIS AND RESULTS}

We evaluate our denoising framework on architectural elements collected at the Seraya site (palace in Turkish) in the ancient city of Nazareth, Israel. Built around 1730, during the Ottoman era, the Seraya served as the regional ruler residence and administrative hub, hence its importance. Over the centuries building parts were added, e.g., a second-floor, characterized by Liwan architectural style. In that respect, the Seraya combines a diverse set of structural elements and details typical to the regional architectural style, evident in the columns, arcs, decorative windows, and type of vaults, and in the different building materials (Fig. 3). The site's exterior was scanned by the Leica C10 TLS from five posts. The upper floor and parts of the internal spaces were scanned using GeoSLAM ZEB-REVO PLS. The registration accuracy of the TLS scans was approx. $3 \mathrm{~mm}$. To register the TLS and PLS clouds, they were first manually coarsely aligned and then an iterative closest point algorithm (ICP) enused, yielding a registration RMSE of $2.1 \mathrm{~cm}$. Overlapping areas between the scans enabled us to compare the fidelity of the denoised point cloud against the TLS counterpart.

The set of parameters for our normal estimation includes the radius, $r$, that defines point cloud neighborhood, $N\left(\mathbf{x}_{i}\right), \eta$ for the robust normal estimation, $\sigma_{n}$ as normal similarity bandwidth for refined normal field, and $\sigma_{r}$ for noise bandwidth and $\sigma_{r_{n}}$ as the projected distance bandwidth. Throughout our experiments, we set $r=3 \mathrm{~cm}$ (same value to $\sigma_{r}$ and $\sigma_{r_{n}}$ ), which is the estimated noise level. This narrow range is justified by the fact that we only need a neighborhood larger than the noise level to determine a plane. We initially set $\eta$ to the maximum of squared 

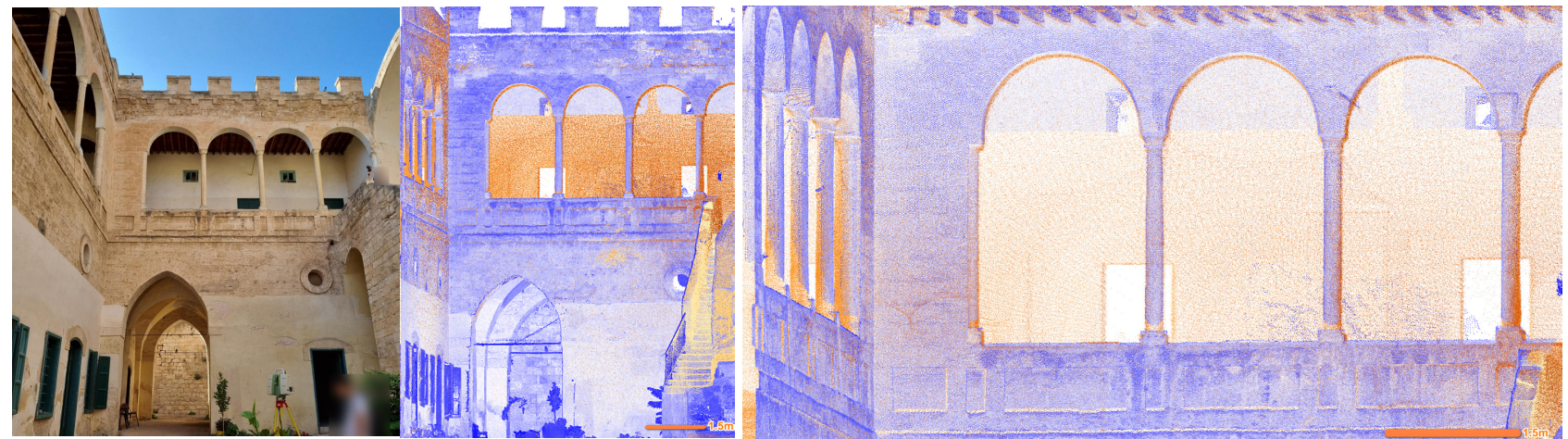

Figure 3. The Seraya site, data collected by an Leica C10 scanner and GeoSLAM Zeb Revo following registration. TLS points are colored in blue, PLS point in orange. For denoising evaluation, we use the TLS data at overlapping area as ground truth.

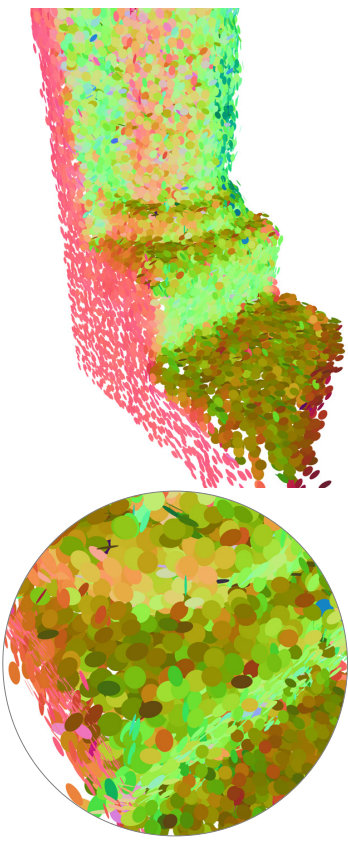

Raw

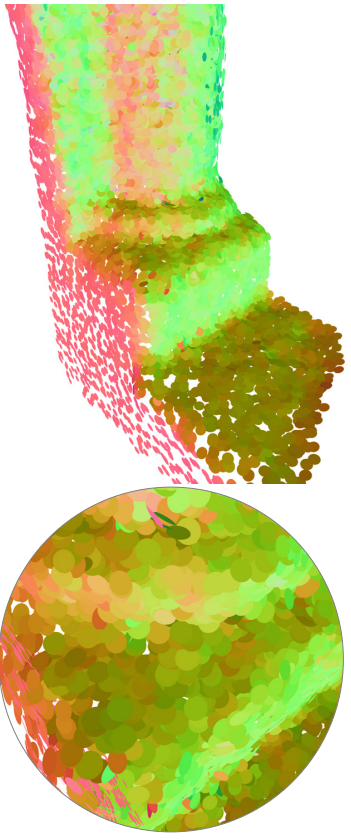

Noise Filter

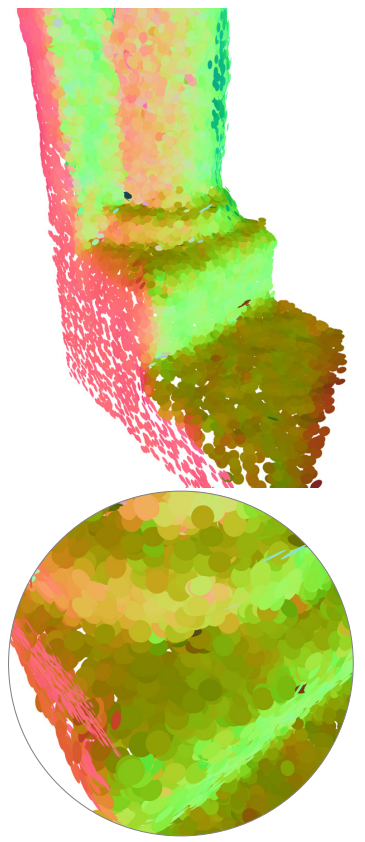

Naive Bilateral

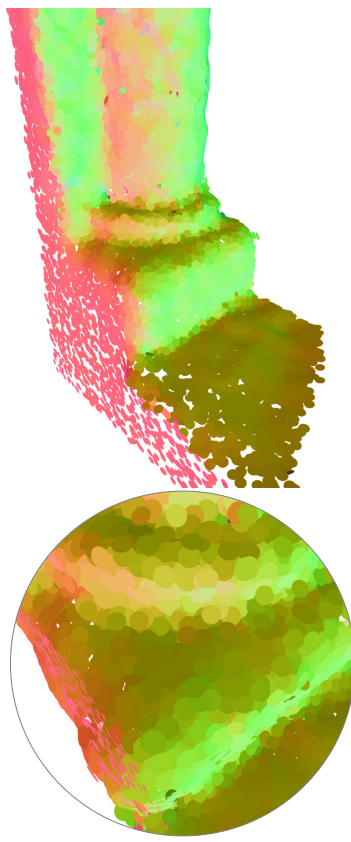

Ours

Figure 4. Comparison of denoising results by using CloudCompare (v.2.11, 2021) noise filter, naive bilateral filter (Digne and De Franchis, 2017), and our approach. Points are colored by their orientations.

projected distances. In addition, $\sigma_{n}$ was set to $15^{\circ}$ throughout our experiment. We fixed their number in all experiments for fair evaluation as a proof of concept, while the outcome of the estimated normals quality can be observed in Fig. (1). We demonstrate how our model produces a well-consolidated point set that preserves sharp features, and how it generates better attributes, e.g., surface normals.

We evaluated our point cloud denoising on a selected set of complex form entities (Figs. 4-6) with comparisons to the plane-fitting based approach, using the CloudCompare (v.2.11, 2021) noise filter implementation, and the direct application of the bilateral filtering approach (Digne and De Franchis, 2017), using the code the authors provide. We firstly show the consolidated pointset of a typical base of a column (Fig. 4), where the cylindrical-shaped column ends in decorative curved support that intersects then a flat planar base. Fig. (4) demonstrates how our approach generates a much smoother output, evident at the transition of surfaces. While the planar- and direct-bilateralfilters failed to generate sharp transitions around intersections of the three geometric forms, over smoothing them, also leaving noisy responses, our approach maintained the salient transitions over the edges while demonstrating smoothness within.

Another indication of the quality of the consolidated pointset and value it may bring is in the generation of smooth normals on challenging geometric setups. To demonstrate this we evaluate its application on a trefoil-pointed arch form, typically found in buildings of the later Ottoman period (Fig. 5). Compared to the results obtained by applying the common noise filter, our solution improves not only the data form but also facilitates smooth normals along the intrados, while maintaining consistency around the pointed tip (Fig. 5).

Finally, we manually selected representative samples of different architectural forms appearing in both PLS and TLS data. Our set consists of decorative structures rich with details, including intersecting curved surfaces, a projecting and recessed brickwork wall pattern that forms a set of parallel planar faces, and a column base rich with decorations. Fig. (6) shows that for all three distinct cases, our model preserves sharp edges, 

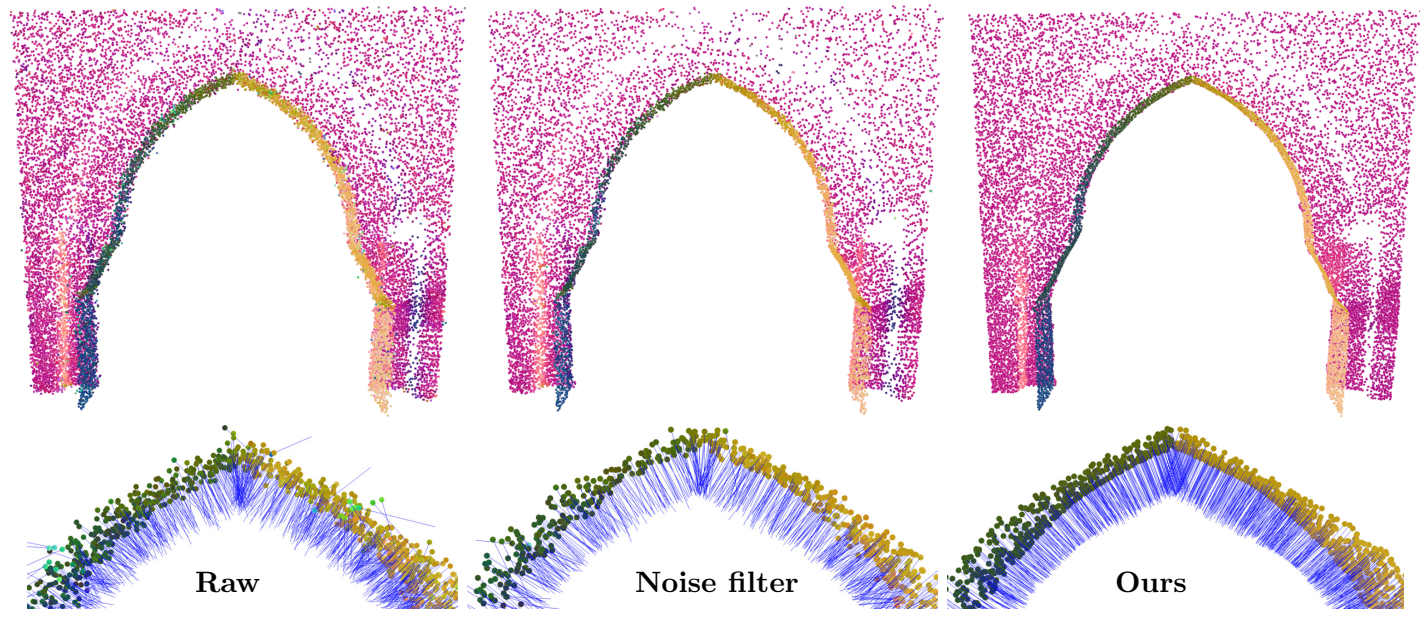

Figure 5. Denoising performed on an trefoil-pointed arch using CloudCompare (v.2.11, 2021) noise filter, and our method. Points are colored by their normals.
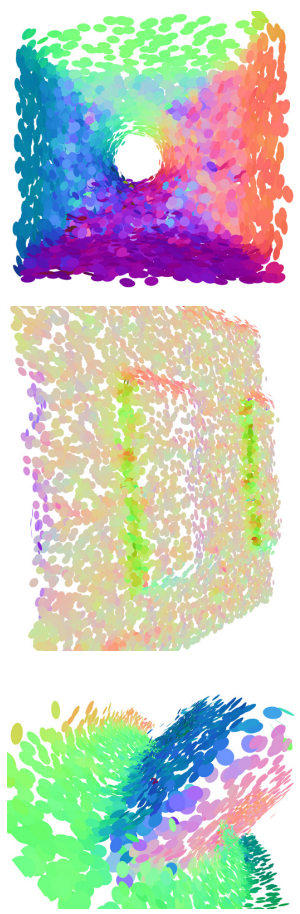

PLS
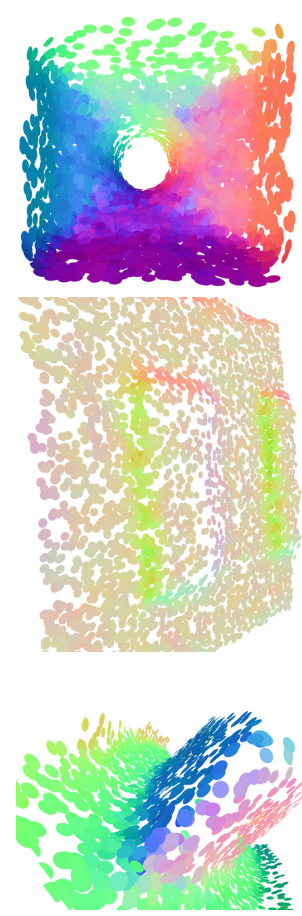

Noise filter
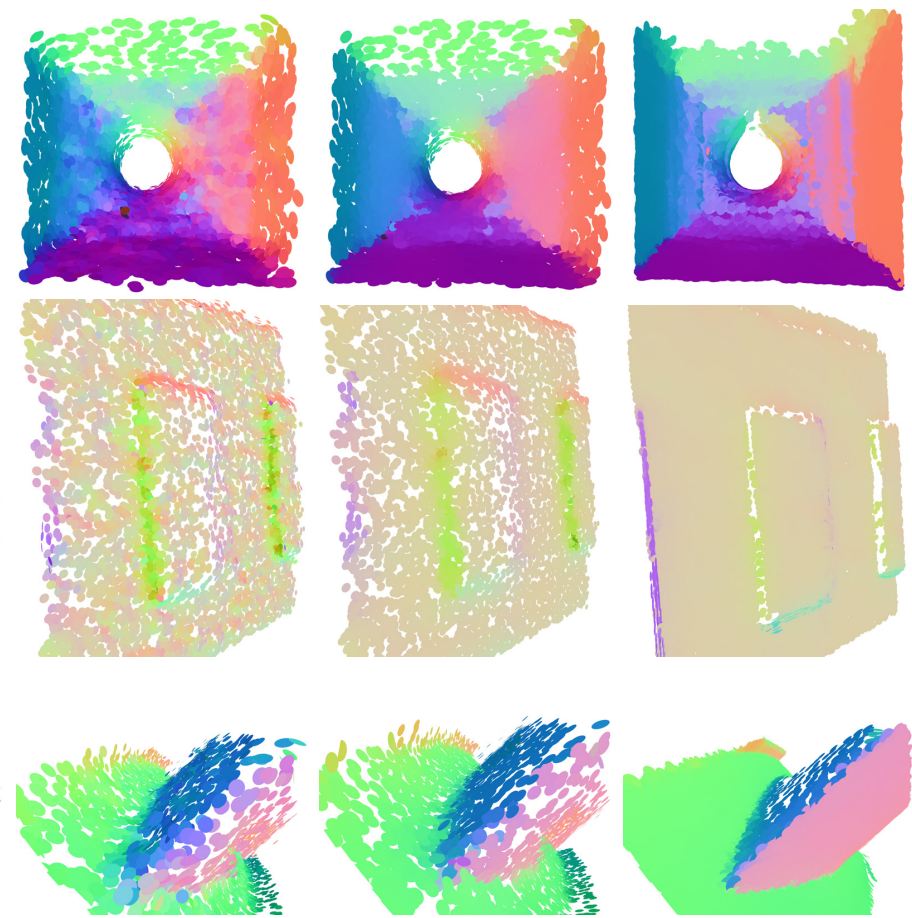

Naive bilateral

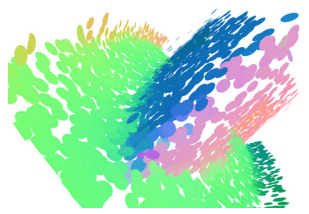

Ours

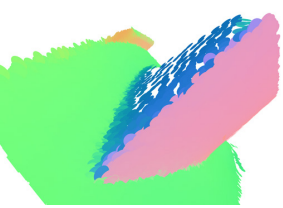

TLS

Figure 6. Noise attenuation using CloudCompare (v.2.11, 2021) noise filter, naive application of the bilateral filter, and our method, compared to TLS data. Points are colored by their normals. Top: A column base (interior view); Middle: projecting and recessed brickwork wall pattern; Bottom: Tip fo a sharp pointed arc.

observable by the intersections of facets and the comparison with TLS data. We close this section with a quantitative analysis of the parallel planes of the projecting and recessed brickwork wall pattern to demonstrate the improvement of attribute estimation by our model. For such a form (Fig. 7), we expect an agreement of normals in local regions, with only minor variations. To quantify this, for each point $\mathbf{x}_{i}$, we analyzed the angular similarity to the normal of each point in its $k$-neighborhood $(k=100)$ and analyzed the distribution of overall normal similarity. While some variations are expected along the edges of the planes, it is expected that most would be similar. As shown in Fig. (7) (note that counts are given in a log scale), our approach produces the outcome with the lowest variations.

\section{CONCLUSIONS}

Realizing that portable laser scanners provide a more efficient documentation technology than their terrestrial counterparts, this paper studied a manner by which the quality of the data can be improved via denoising of such point clouds. Recognizing that prevalent methods are mostly planar-surface-based, its objectives were to handle the high level of noise, as well as preserve structure, especially around discontinuous surfaces. Recognizing that local surface normals play a pivotal role in such a computation, it first applied a robust approach to their 
Test Model
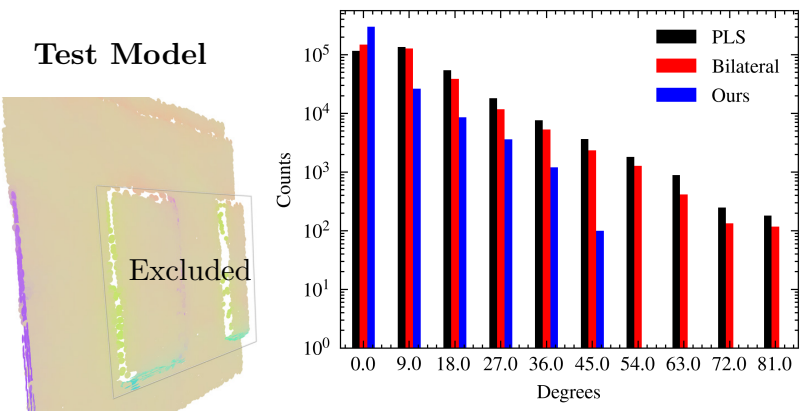

Figure 7. Study of estimated normal variations using the naive bilateral filter and our approach on projecting and recessed brickwork wall pattern, excluding marked regions.

computation and then improved their quality by iteratively reweighting nearby points by their present normal value. This way, the effect of surfaces not related to the analyzed point, was limited. In order to further limit the local planarity assumption, we cast the denoising solution into the bilateral filtering framework. This has further reduced the effect of non-related points of different surfaces, and improved the preservation of the underlying surface shape. Results demonstrate the contribution of our approach in enhancing the quality of portable laser scans, allowing us to highlight details and obtain more consistent surface features. This, in turn, may help in subsequent processing, in either segmentation- or visualization-related applications.

\section{ACKNOWLEDGMENTS}

The authors would thank the Nazareth old city committee and the Nazareth municipality for their support.

\section{References}

Bronzino, G., Grasso, N., Matrone, F., Osello, A., Piras, M., 2019. Laser-visual-inertial odometry based solution for 3D heritage modeling: the sanctuary of the blessed virgin of trompone. International Archives of the Photogrammetry, Remote Sensing \& Spatial Information Sciences.

Capone, M., Lanzara, E., 2019. 3D data interpretation using treatises geometric rules to built coffered domes. International Archives of the Photogrammetry, Remote Sensing \& Spatial Information Sciences.

Cui, Y., Li, Q., Yang, B., Xiao, W., Chen, C., Dong, Z., 2019. Automatic 3-D reconstruction of indoor environment with mobile laser scanning point clouds. IEEE Journal of Selected Topics in Applied Earth Observations and Remote Sensing, 12(8), 3117-3130.

Digne, J., De Franchis, C., 2017. The bilateral filter for point clouds. Image Processing On Line, 7, 278-287.

Dinesh, C., Cheung, G., Bajić, I. V., 2020. Point cloud denoising via feature graph laplacian regularization. IEEE Transactions on Image Processing, 29, 4143-4158.

Huang, H., Wu, S., Gong, M., Cohen-Or, D., Ascher, U., Zhang, H., 2013. Edge-aware point set resampling. ACM transactions on graphics (TOG), 32(1), 1-12.
Kopp, J., 2008. Efficient numerical diagonalization of hermitian $3 \times 3$ matrices. International Journal of Modern Physics $C$, 19(03), 523-548.

Liu, Z., Xiao, X., Zhong, S., Wang, W., Li, Y., Zhang, L., Xie, Z., 2020. A feature-preserving framework for point cloud denoising. Computer-Aided Design, 127, 102857

López, F. J., Lerones, P. M., Llamas, J., Gómez-GarcíaBermejo, J., Zalama, E., 2017. A framework for using point cloud data of heritage buildings toward geometry modeling in a BIM context: A case study on Santa Maria La Real De Mave Church. International Journal of Architectural Heritage, 11(7), 965-986.

Murtiyoso, A., Grussenmeyer, P., 2019. Automatic heritage building point cloud segmentation and classification using geometrical rules. International Archives of the Photogrammetry, Remote Sensing \& Spatial Information Sciences.

Ning, X., Li, F., Tian, G., Wang, Y., 2018. An efficient outlier removal method for scattered point cloud data. PloS one, 13(8), e0201280.

Nurunnabi, A., West, G., Belton, D., 2015. Outlier detection and robust normal-curvature estimation in mobile laser scanning 3D point cloud data. Pattern Recognition, 48(4), 14041419.

Orts-Escolano, S., Morell, V., García-Rodríguez, J., Cazorla, M., 2013. Point cloud data filtering and downsampling using growing neural gas. The 2013 International Joint Conference on Neural Networks (IJCNN), IEEE, 1-8.

Patrucco, G., Rinaudo, F., Spreafico, A., 2019. Multi-source approaches for complex architecture documentation: the" Palazzo Ducale" in Gubbio (Perugia, Italy). International Archives of the Photogrammetry, Remote Sensing \& Spatial Information Sciences.

Russhakim, N., Ariff, M., Majid, Z., Idris, K., Darwin, N., Abbas, M., Zainuddin, K., Yusoff, A., 2019. The suitability of terrestrial laser scanning for building survey and mapping applications. International Archives of the Photogrammetry, Remote Sensing \& Spatial Information Sciences.

Sanchez, J., Denis, F., Coeurjolly, D., Dupont, F., Trassoudaine, L., Checchin, P., 2020. Robust normal vector estimation in 3D point clouds through iterative principal component analysis. ISPRS Journal of Photogrammetry and Remote Sensing, 163, 18-35.

Wang, C., Wen, C., Dai, Y., Yu, S., Liu, M., 2020. Urban 3D modeling with mobile laser scanning: a review. Virtual Reality \& Intelligent Hardware, 2(3), 175-212.

Xia, S., Chen, D., Wang, R., Li, J., Zhang, X., 2020. Geometric primitives in LiDAR point clouds: A review. IEEE Journal of Selected Topics in Applied Earth Observations and Remote Sensing, 13, 685-707.

Xie, L., Wang, R., 2017. Automatic indoor building reconstruction from mobile laser scanning data. International Archives of the Photogrammetry, Remote Sensing \& Spatial Information Sciences, 42.

Zeng, J., Cheung, G., Ng, M., Pang, J., Yang, C., 2019. 3D point cloud denoising using graph Laplacian regularization of a low dimensional manifold model. IEEE Transactions on Image Processing, 29, 3474-3489. 